\title{
VALUE SENSITIVE DESIGN IN THE DEVELOPMENT OF DRIVERLESS VEHICLES: A CASE STUDY ON AN AUTONOMOUS FAMILY VEHICLE
}

\author{
R. Graubohm ${ }^{\otimes}$, T. Schräder and M. Maurer \\ Technische Universität Braunschweig, Germany \\ $\bowtie$ graubohm@ifr.ing.tu-bs.de
}

\begin{abstract}
Complex new functionalities and dissimilar stakeholder groups pose challenges to the requirement analysis for driverless vehicles. To overcome these challenges, we propose a value-oriented reference process for innovative functionalities of an autonomous family vehicle. The valueoriented measures are taken from the approach of Value Sensitive Design. In our application, we have found that the consideration of the human values involved is of great importance for the identification of stakeholders and the management of their potentially conflicting interests throughout the development process.
\end{abstract}

Keywords: driverless vehicles, case study, value sensitive design, early design phase, product development

\section{Introduction}

Disruptive vehicle concepts and autonomous driving functions require novel development processes to address the wide range of involved aspects. The autonomous family vehicle autoELF displays a new mobility use case and an unprecedented functional range. The challenge to create a sound set of requirements for a new application demands a comprehensive use of design approaches and methods. Approaches for incorporating human values in the design of technology are of major interest in industry and academia, since societal acceptance is a prerequisite for a product's success. We therefore aim at applying methods for the consideration of human values throughout the development process.

Value Sensitive Design is a methodology to investigate and integrate values into systems that originated from information technology (Friedman et al., 2013). The wide definition and thorough analysis of stakeholders and their potentially conflicting interests with regard to a system's functionality are major characteristics of the methodology. With respect to the functional range of automated vehicles, the particular perspective taken in Value Sensitive Design can be of remarkable interest in order to align design decisions with affected values of different stakeholders.

In this paper, we apply Value Sensitive Design looking at the fulfillment of subsidiary tasks in an autonomous family vehicle. Subsidiary tasks are tasks that drivers today accomplish in direct interaction with passengers in addition to the driving task (Schräder et al., 2019). Examples for subsidiary tasks are the monitoring of passengers' safe entries into and exits from the vehicle, the coordination of travel requests, the supervision and safeguarding of minors, etc. If performed by human drivers while driving, subsidiary tasks can also be characterised as tertiary tasks (Bubb, 2007) or non-driving-related activities (Pfleging et al., 2016). Mostly, these tasks are related to safety, 
comfort, and accessibility of different user types and their fulfillment is indispensable. Consequently, providing functions which fulfill subsidiary tasks is of major interest for the use case of a multigeneration family vehicle.

The functional specification for the innovative cases of application provided by the vehicle concept raises novel problems. Due to the lack of experience from similar applications, customer expectations and conflicts of interest between different stakeholder groups are widely unknown. We thus use a value-oriented design approach to compensate for the missing practical experience and best practices with regard to the system under development.

The methodology and the project context are explained in the following sections. Subsequently, we describe how we plan to include human values into the system design by mapping Value Sensitive Design to a development process outline. Finally, the application and results of early considerations of Value Sensitive Design methods for requirement analysis are presented.

\section{Value sensitive design}

Based on previous work, Friedman et al. describe Value Sensitive Design as a "theoretically grounded approach to the design of technology that accounts for human values in a principled and comprehensive manner throughout the design process" (Friedman et al., 2013, p. 56). They state that the approach "adopts the position that technologies in general [...] provide value suitabilities that follow from properties of the technology" (Friedman et al., 2013, p. 61). Detailing the methodology, Friedman et al. (2013) introduce three types of investigations performed integratively and iteratively during a system's design. Essentially, Value Sensitive Design adds value-oriented investigations to individual phases of a development without necessarily changing the overall process (e.g., the Vmodel of development).

The term value is defined in Value Sensitive Design as something that "a person or group consider important in life" (Friedman et al., 2013, p. 57). Friedman et al. (2013) describe the iterative conduction of investigations as the basis to identify stakeholders and their values and incorporate values in the system design. In their methodology, stakeholders are groups, organizations, and individuals directly and indirectly affected by the product under development. The following paragraphs describe the three types of investigations by Friedman et al. (2013) and Friedman et al. (2017): conceptual investigations, technical investigations, and empirical investigations.

\subsection{Conceptual investigations}

Beginning in early design stages, experts perform conceptual investigations to identify all stakeholders directly and indirectly affected by the intended functional scope of the system under development. The investigations aim to uncover values, value trade-offs, as well as value hierarchies. A specific goal of the investigations is the conceptualization of specific values for reasons of clarification and for later reference.

\subsection{Technical investigations}

Technical investigations are analyses of the impact of specific technological properties and mechanisms on value suitabilities and value trade-offs. Furthermore, these analyses include the identification of system designs that are capable of addressing stakeholders' values.

\subsection{Empirical investigations}

Empirical investigations are applications of qualitative and quantitative methods from social science research to augment and validate value analyses. Designers use empirical studies not only to legitimize the lists of stakeholders and values affected but also to evaluate whether solutions capture specific values as intended. An important piece of information from the empirical methods is the investigation of the stakeholders' prioritization of values in trade-off situations.

Empirical studies are thoroughly useful for validation within value-oriented development processes. Specifically, concluding empirical investigations are an important instrument to ensure that all relevant values were sufficiently incorporated into a technical solution. The aspect of evaluating stakeholders' approval at the end of development iterations is further highlighted in the reference 
process presented in Section 4. Before detailing our approach, we briefly introduce our case of application and the project context in the following section.

\section{Project context}

The development of an autonomous family vehicle is one of the goals of the research project UNICARagil, funded by the German Federal Ministry of Education and Research (BMBF). The vehicle concept and its prototypical realization are named autoELF, which refers to a mythological figure that performs household tasks in the service of a family.

The four UNICARagil vehicles are based on a common modular platform, but are designed for diverse use cases. In addition to the autonomous family vehicle autoELF, an autoTAXI, an autoSHUTTLE, and an autoCARGO will be realized (cf. Woopen et al. (2018) for a detailed use case description). The project consortium of institutes from eight German universities is aiming to showcase all four driverless cars by 2022. Six industrial partners support the development. One distinct feature of the UNICARagil system, which will also be prototypically realized, is the control room that complements the driverless vehicles (Woopen et al., 2018). Operators in the control room will be able to communicate with passengers and remotely operate the vehicles in cases of interruptions of normal operation.

In previous work (Schräder et al., 2019), we started to systematically identify functional requirements for the autonomous family vehicle autoELF by analyzing subsidiary tasks accomplished by accompanying persons in conventional cars. A list of required functional competencies of the vehicle was inferred from use case diagrams. Figure 1 illustrates three identified key vehicle functions of an autonomous family vehicle: health monitoring, remote trip planning, and barrier-free access.

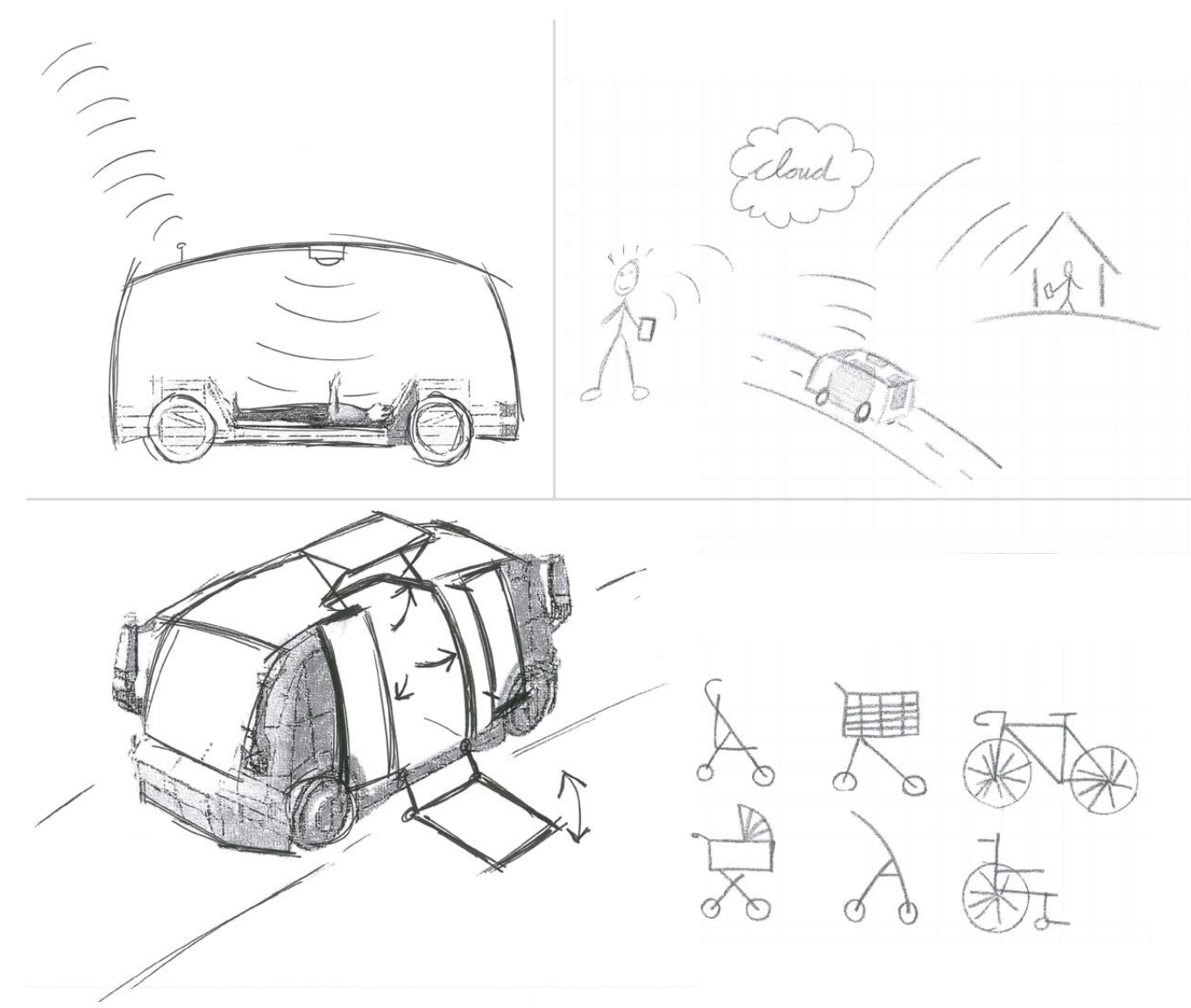

Figure 1. Illustrations of required vehicle functions: medical emergency measures (top left), shared web-based travel planning (top right), barrier-free access (bottom)

The illustrated vehicle functions are part of the value-oriented development approach described in the following sections. In addition, we consider the vehicle's competencies to select suitable entry and exit 
spots, to grant individual users' rights, to report passengers' misbehavior, and to prevent use by unknown persons. The introduction of human values affected and captured by technological functionalities provides a basis for a revision of functional and non-functional requirements. Uncovered values can serve as both, a support for design decision or novel constraints of the solution space.

In the following section, we describe the integration of further investigations in a reference process for the iterative development of automotive systems presented in Graubohm et al. (2017). Subsequently, we apply Value Sensitive Design methods in an early stage of development for the interior features of the autonomous family vehicle.

\section{Integrating value-oriented investigations in a reference development process of automated vehicles}

In previous work (Graubohm et al., 2017), we described the development of automated vehicles by displaying two distinct cycles: an inner cycle that is purely conceptual and an outer cycle that comprises concrete system design, (prototypical) implementation, and testing. The development process outline was mainly influenced by safety-driven design experiences in early development stages. Figure 2 displays a mapping of the value-oriented design investigations presented in Section 2 to the course of development of an automated vehicle.

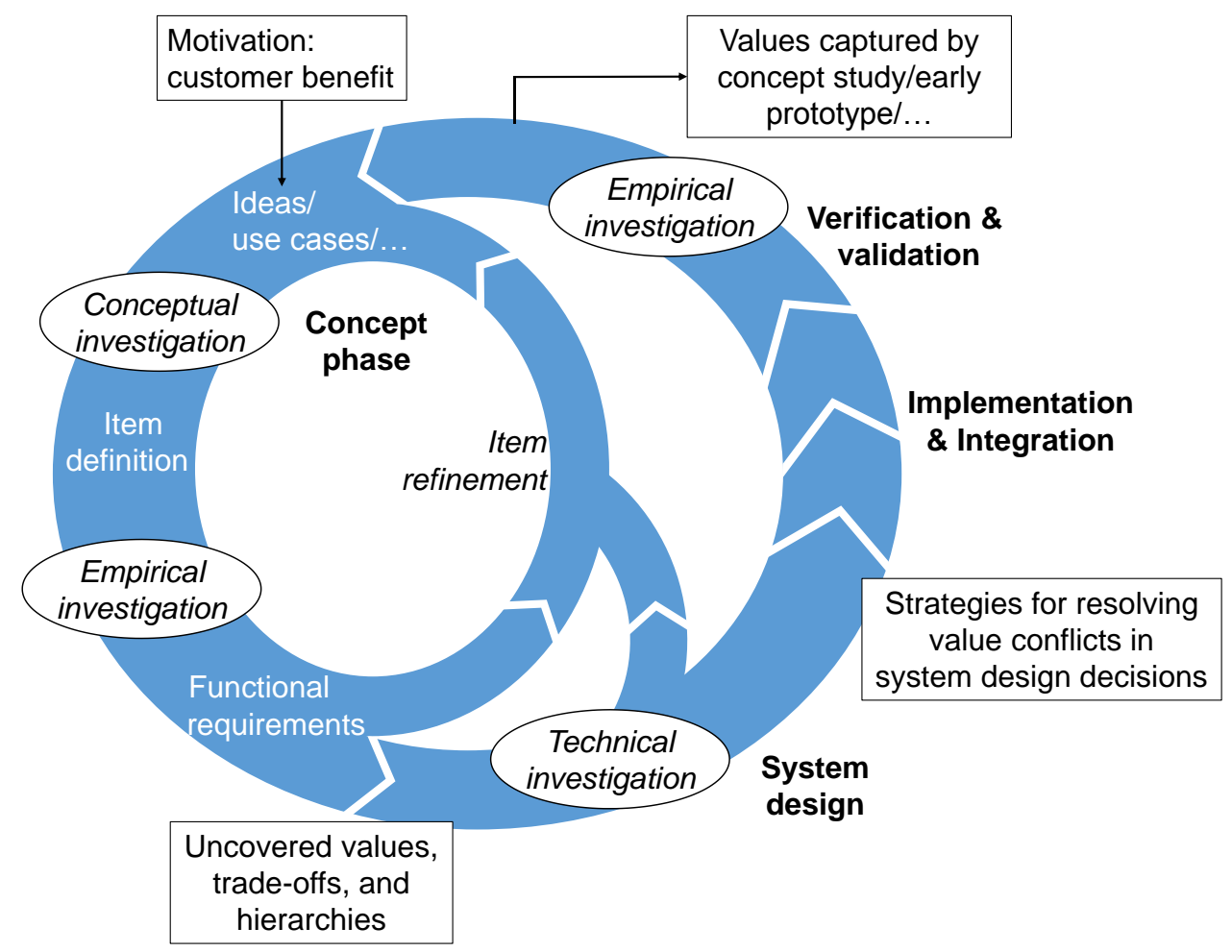

Figure 2. Value sensitive design investigations mapped to the reference process outline proposed in Graubohm et al. (2017)

The process outline begins with the motivation to address an identified customer benefit, and every full iteration of this outline results in the creation of concept studies, prototypes, or complete product specifications. In order to find and capture values of all direct and indirect stakeholders in these development artifacts, we propose the introduction of all three types of investigation defined in the Value Sensitive Design methodology: conceptual, technical, and empirical investigations.

In the concept phase of development, customer benefits are expressed in a concrete description of functionality, i.e. "item definition", on the basis of ideas, use cases, etc. Subsequently, functional requirements addressing all contents of the item definition have to be specified. We use the term 
"functional requirement" for a formal specification of a demanded system functionality that can address any design aspect, including safety. In the concept stage, functional requirements are expressed independent of the concrete technological implementation. The goal of the introduction of Value Sensitive Design into the concept phase is the knowledge of relevant values, hierarchies, and trade-offs in addition to the functional specification when initializing the concrete system design in the next phase. The inner loop, denoted "item refinement", expresses the need for an adaptation of the functional specification after design conflicts occur that cannot be resolved with measures of technical system design. Reasons for design conflicts can be safety considerations, marketing aspects, but also human value investigations. Particularly, uncovered value tensions and value conflicts between different stakeholders can induce unresolvable antitheses that require the functional description to be adapted.

We propose to introduce conceptual investigations and subsequent empirical investigations in the concept phase of automated vehicles. With the aim to identify stakeholders and their values, conceptual investigations can be a valuable input for the item definition. Nonetheless, subsequent empirical investigations are generally required to validate the assumptions made by designers within conceptual investigations. Based on the detailed description of potential functionalities within the item definition empirical investigations legitimize the results of conceptual investigations and determine value hierarchies. The development of medical emergency measures for an autonomous vehicle (cf. Figure 1) can be used as an example to explain the sequence of two investigation types. In principle, the functionality of health monitors aims to satisfy the passengers' interest in physical welfare. Analyzing potentially impaired values in conceptual investigations designers will be able to identify privacy concerns related to the introduction of health monitoring. Consequently, empirical investigations are needed to prove the presence of privacy concerns and investigate their importance for target users.

If assumptions of the conceptual investigation are contradicted by the results of empirical investigations, an iteration is required within the concept phase, depicted by the inner cycle. The empirical evidence of involved values and present hierarchies is subsequently expressed in functional requirements. For this, abstract value-related descriptions have to be transferred into formal language that allows an implementation. E.g., the decision "health data will not be stored because of privacy concerns over the storage of data in the vehicle" has to be translated into "the system shall delete health data of passengers after their ride is completed".

After a consistent specification of functionality expressed in a sound set of requirements is identified and documented, the subsequent stage of system design can be performed. The system design phase concretizes functionality by specifying technologies utilized and by expressing the design specification in concrete technical requirements that serve as an input for domain-specific implementation (e.g., hardware and software implementation). In addition, we propose to specify strategies for resolving value conflicts as a goal of the system design.

The major input for value considerations in the system design stage are technical investigations. On the one hand, technical investigations can help to identify implementation strategies that fit the need of addressing known value trade-offs. On the other hand, technical investigations can be a basis for ensuring that selected implementation strategies are not in conflict with known stakeholder values.

For the example of health monitoring in an automated vehicle, designated sensors and data processing approaches have to be analyzed under consideration of impaired values like privacy. Moreover, a value-related design conflict is present, if empirical evidence indicates that the importance of privacy versus physical welfare is different between user types. A technical solution to resolve the conflict is disabling the system by default and prompting new users to actively agree to health monitoring.

Figure 2 displays the option of an item refinement initiated in the system design stage. One cause for the need of an immediate adaptation of the functional specification can be the outcome of technical investigations: if known and available technology lacks the potential to address uncovered stakeholder values, the desired functional range has to be adjusted.

Empirical investigations have already been performed in the concept phase, but are again incorporated in the verification and validation phase of our process outline. Concluding the outer cycle, feedback on prototypes, simulations, or early samples of vehicle functionalities can be obtained from potential users and other stakeholders. These investigations validate whether the uncovered values are captured as intended by the design decisions. 


\section{Use case of an autonomous family vehicle}

The common modular platform of the different prototypes of the research project UNICARagil (comprising frame, driving actuators, environment perception system, etc.) is already in the stage of implementation. However, the development process of the individual characteristics of the autonomous family vehicle autoELF (cf. Section 3) can still be considered in the concept stage. So far, the vehicle functionalities for fulfilling subsidiary tasks, providing safety, comfort, and accessibility for users of the system have already been expressed in early functional requirements. With regard to the reference process discussed in the previous section, the development of these vehicle functionalities passed the stages of the concept phase at least once: a functional specification and requirements were produced by investigating the needs of the user in specific use cases (cf. Schräder et al., 2019). However, the requirement elicitation so far was solely based on the perspective of passengers in few specific scenarios. Due to the innovative character of autoELF, we chose a value-oriented design approach to systematically investigate and explain customer expectations and conflicts of interest between different stakeholder groups. During our early requirement analysis for the vehicle concept, we found that many stakeholder values have to be respected and integrated into technological solutions beyond the act of driving. In our use case, accessibility is essential for the target user group and current reports indicate that individual passenger characteristics are also increasingly important in commercial autonomous mobility projects. Waymo, for example, integrated design elements in their vehicles to meet the needs of the elderly and individuals with disabilities (Halsey, 2017).

In a new iteration of the concept phase (i.e. item refinement), we aim to perform the value-oriented investigations illustrated in Figure 2. Our goal is to find additional requirements and constraints of our solution space. Additionally, we want to obtain knowledge about all involved stakeholders, their values, and present value conflicts as an input for the system design and prototypical implementation.

As a first step of the new iteration of our requirement analysis, we consider the use cases already discussed in the previous publication: entry, exit, and ride scenarios with unaccompanied children or elderly people as passengers. So far, the evaluation of these scenarios was focused on the needs and desires of passengers. In contrast, we now try to systematically list stakeholders and identify involved human values (i.e. conceptual investigation) before further specifying functionalities and requirements.

With the aim to find new aspects for the prototypical implementation of the vehicle concept, we limit the input for the conceptual investigation to the design team, without involving experts in psychology. However, for a mature product development with the aim to release a vehicle to the market, external consultation would definitely be needed.

\subsection{First results}

In addition to passengers that are the direct user and stakeholder of the designed functions, we identified specific direct and indirect stakeholder groups for functionalities of the family vehicle. The values of these stakeholders were investigated with regard to our functional specification and preliminary system design. In contrast to earlier considerations, the functional specification and use cases now invoke additional stakeholders that were not displayed in the use case diagram of previous work. As an example, we now consider developers as a relevant stakeholder group and further analyze control room operators' interests and values as a fallback and communication channel with the passengers in cases of interruptions of normal operation (Woopen et al., 2018). The list of affected stakeholders includes:

- Users

- Developers

- Entrepreneurs

- Maintainers

- Operators (in control rooms)

- Mobility service providers

- Traffic participants

- Environmental associations

- Politicians

- Type-approval authorities if required by law (e.g. EU) 
Considering the special use case of an autonomous family vehicle, we additionally split the stakeholder group "users" into the passengers and indirect users (i.e. other family members). The activities and involved values with regard to the fulfillment of subsidiary tasks in autoELF are dissimilar for these two groups. Adult family members that own a driver license and are capable of driving a car are not the typical passenger in the use cases for the new vehicle. They will mainly interact with autoELF in order to plan and supervise the rides of young or elderly family members or cargo (e.g. groceries).

Other listed stakeholders are mainly relevant for specifying the driving function of the autonomous vehicle. Functionalities that potentially interfere with traffic (e.g., the decision for an acceptable stop location and door opening) depend on the driving function. Thus, when considering nondriving related safety, comfort, and accessibility functions, the interference with other traffic participants is insignificant. Table 1 provides an overview of human values of the most important stakeholder groups.

Table 1. Overview of stakeholder groups and involved values

\begin{tabular}{ll}
\hline Stakeholders & Values \\
\hline Passengers & $\begin{array}{l}\text { Safety, mobility, freedom, security, aesthetics, haptics, efficiency, trust, privacy, } \\
\text { sustainability, accessibility, ownership }\end{array}$ \\
\hline Indirect users & Safety, mobility, security, efficiency, trust, privacy, sustainability, accessibility, ownership \\
\hline Developers & Safety, mobility, trust, sustainability, traceability, transparency \\
\hline Maintainers & Sustainability, transparency, simplicity \\
\hline Operators & Safety, mobility, efficiency, trust, sustainability, transparency, simplicity \\
\hline
\end{tabular}

With regard to the conceptualization of the value categories, we generally adopted the definitions provided by Friedman et al. (2013). However, safety is specifically chosen over the abstract human welfare category in order to express the major concern of most potential users in interaction with driverless vehicles. Based on our experience, efficiency was also added as a value expressing both, the interest in time and cost efficiency, where weighting is different for individual stakeholder groups.

Almost all stakeholder groups share the objective of trust. The definition of trust provided by Friedman et al. (2013) for applying Value Sensitive Design is based on an earlier publication on web applications (Friedman et al., 2000) and has limitations in its applicability to automated vehicles. We therefore adopted the conceptualization of trust in automation introduced by Lee and See (2004) and further analyzed by Hoff and Bashir (2015). Following Lee and See, trust is "the attitude that an agent will help achieve an individual's goals in a situation characterized by uncertainty and vulnerability" (Lee and See, 2004, p. 54). Thus, from the perspective of a passenger, trust in autoELF is a prerequisite for calmness and a firm belief in sufficient safety. Moreover, satisfying traditional design criteria like reliability supports building trust in the automated vehicle.

As an additional result of conceptual investigations, we can already identify emerging value conflicts. For individual users, the vehicle design will have to be a trade-off between the goals of mobility, efficiency, freedom on the one hand and safety on the other hand. Also, since the control room is part of the vehicle concept in order to increase robustness of the functionalities (Woopen et al., 2018), meeting privacy and mobility demands of the passengers is an emerging trade-off. Value conflicts between the different stakeholder groups can be illustrated by the example of the developers' and operators' interest in vast transparency and the users' interest in privacy.

Lastly, hierarchies between conflicting values can be predicted before validating the considerations in subsequent empirical investigations described in the following section. As an example, the passengers' interest in safety should generally come before haptics and aesthetics.

In order to prepare further value-oriented investigations, we assessed the relations of values identified in conceptual investigations with the functional specification of the subsidiary tasks in a family vehicle described in Schräder et al. (2019). The combination of detailed functional descriptions and involved values corresponds with iterating the "item definition" named in Figure 2. Some examples of the documented correlations are presented in Table 2. 
Table 2. Examples of vehicle functionalities and affected values

\begin{tabular}{|c|c|c|c|}
\hline Vehicle function & Satisfied values & Impaired values & Ambiguity \\
\hline $\begin{array}{l}\text { Allowing entries and exits only at } \\
\text { suitable spots }\end{array}$ & $\begin{array}{l}\text { Safety, accessibility, } \\
\text { simplicity, traceability }\end{array}$ & $\begin{array}{l}\text { Mobility, freedom, } \\
\text { efficiency }\end{array}$ & Trust \\
\hline $\begin{array}{l}\text { Limiting users' rights for specific } \\
\text { passengers (e.g. minors) }\end{array}$ & Safety, trust & $\begin{array}{l}\text { Mobility, freedom, } \\
\text { privacy }\end{array}$ & \\
\hline $\begin{array}{l}\text { Reporting of and reacting to } \\
\text { passengers' misbehavior }\end{array}$ & Safety, transparency & $\begin{array}{l}\text { Mobility, freedom, } \\
\text { efficiency, privacy }\end{array}$ & Trust \\
\hline $\begin{array}{l}\text { Option for all family members to } \\
\text { order the vehicle, plan trips, and } \\
\text { control HMI functions }\end{array}$ & $\begin{array}{l}\text { Mobility, freedom, trust, } \\
\text { accessibility, ownership, } \\
\text { transparency }\end{array}$ & Safety, efficiency & Traceability \\
\hline $\begin{array}{l}\text { Support for loading and securing } \\
\text { wheelchairs, luggage, etc. }\end{array}$ & $\begin{array}{l}\text { Safety, mobility, freedom, } \\
\text { trust, accessibility, } \\
\text { sustainability }\end{array}$ & Aesthetics & \\
\hline $\begin{array}{l}\text { Prevention of entry and use by } \\
\text { unknown persons }\end{array}$ & Security, ownership & $\begin{array}{l}\text { Mobility, freedom, } \\
\text { privacy, } \\
\text { accessibility }\end{array}$ & Trust \\
\hline $\begin{array}{l}\text { Video supervision of the vehicle's } \\
\text { interior }\end{array}$ & $\begin{array}{l}\text { Safety, efficiency, } \\
\text { traceability, transparency }\end{array}$ & $\begin{array}{l}\text { Freedom, trust, } \\
\text { privacy, ownership }\end{array}$ & \\
\hline
\end{tabular}

The first vehicle function reflected in the table is the limitation of autoELF to only initiate entry and exit procedures in positions identified as suitable by the driving function. The evaluation can be based on map and environment data and considers safety aspects. Additionally, by choosing suitable entry and exit spots, the fulfilment of the users' needs with regard to accessibility can be ensured. Yet, the autonomy of individual passengers to decide where to enter and exit the vehicle is restricted, impairing the individuals' values of mobility and freedom. Also, we found the effect of this function on the stakeholder value of trust to be ambiguous. On the one hand, the vehicle avoids putting its users into hazardous situations during entry and exit. Furthermore, it ensures usability for the current passengers (e.g., by choosing spots where systems for barrier-free access can be used). This could lead to a satisfaction of the trust value on the user side. On the other hand, passengers might also encounter scenarios where the vehicle unforeseeably decides against a close stopping position. The potential of feeling trapped in the vehicle until it finds a suitable holding spot can likely impair the perceived trust. Another vehicle function listed in Table 2 reflects the idea of unequal users' rights for vehicle functionalities. One example discussed in Schräder et al. (2019) is the prevention of minors to change the destination by using the interior HMI. The satisfied values (safety, trust, etc.) are mostly displayed by parents that would be the indirect user in the scenario, while the values of the children that are the actual passengers are highly impaired (mobility, freedom, etc.).

\subsection{Outlook}

The next value-oriented process step of our outline (cf. Figure 2) are empirical investigations. Friedman et al. present semi-structured interviews as an advantageous method for stakeholder consultation in an early design stage: "A semi-structured interview often offers a good balance between addressing the questions of interest and gathering new and unexpected insights" (Friedman et al., 2017, p. 99). With the objective to understand the stakeholders' judgement about value conflict occurring in the context of use, we plan to interview representatives of the stakeholder groups identified in the previous section.

Subsequently, present design conflicts will be disclosed and addressed in functional requirements before initiating the system design phase of functions which fulfill subsidiary tasks in autoELF. We intend to perform technical investigations in a continuous and iterative manner in this phase to monitor the influence of technological decisions on the uncovered values. The overall objective will be to meet the stakeholders' attitude towards trade-off situations with our design decisions. However, technological investigations of Value Sensitive Design do not only focus on ways to respect value trade-offs during implementation, but also advise designers to find solutions for later adoption to a changed value hierarchy in specific applications. For example, the family mobile application that serves as an interface 
for individual family members to the vehicle can offer protected individual settings regarding vehicle tracking and interior video supervision. These settings would allow the user group of a particular vehicle (i.e. a specific family) to adapt the extent of remote surveillance to their individual value hierarchy.

Lastly, we intend to realize many design elements for critical functionalities of an autonomous family vehicle in the prototype of autoELF. Empirical studies using the prototype will enable us to validate whether our solution succeeds in meeting the relevant human values.

\section{Related work}

Due to their roots in information technology, few publications apply the value-oriented investigations of Value Sensitive Design within the development of mechatronic systems in the transportation domain. Most use cases discussed as examples are focusing on software design, often with emerging privacy conflicts (Davis and Nathan, 2015; Friedman et al., 2013; Friedman et al., 2017; Winkler and Spiekermann, 2018).

Cawthorne and Cenci (2019) apply Value Sensitive Design to analyze a prototype of a humanitarian cargo drone. They perform all three types of investigations in part and present an alteration of the system design that aims at meeting uncovered values of human welfare and sustainability. Within their conceptual analysis, Cawthorne and Cenci find that the objective to incorporate human values in a technological design can be strongly linked with several of the United Nations Sustainable Development Goals (UN SDGs) (Cawthorne and Cenci, 2019, p. 1120).

Gerdes et al. (2019) describe an approach of facilitating value-based conversations in engineering environments. They name Value Sensitive Design as a major inspiration. Gerdes et al. reason their valuecentered approach by value conflicts of safety, mobility, and legality during the design of algorithms for the driving tasks. The integration of value considerations into algorithms for behavior decisions for automated vehicles is presented in detail in two publications by Thornton (2018) and Thornton et al. (2018).

Within their value-centered approach, Gerdes et al. (2019) face the problem that human values are not a common language in discussions of engineering problems. Gerdes et al. identify the need for tools and methods to establish human values as a common terminology in order to allow interdisciplinary analyses including many stakeholder groups in design processes. In addition, Gerdes et al. emphasize the importance of mapping design parameters to values, enabling engineers to argue how individual values are captured in the system.

The related work indicates a growing importance of value-oriented approaches for innovative and automated transportation applications. In this context, we aim to contribute to a better understanding of how to perform value analyses throughout the development of automated vehicles by introducing a reference process and a new use case.

\section{Conclusion}

The main objective of our work is to systematically include stakeholder groups and their interests in the requirement analysis for development processes of automated vehicles. One possible approach is the consideration of human values during mechatronic system design. In order to realize value considerations, we integrated Value Sensitive Design into an existing process outline for the development of automated vehicles.

As part of the research project UNICARagil, we applied the proposed development process in the case study of fulfillment of subsidiary tasks in the autonomous family vehicle autoELF. We presented the use of value-oriented investigations in the early design stage and obtained a list of critical stakeholders and current conflicts of interest in our use case. The method increased our awareness of the implications of the innovative nature of the vehicle project: besides criteria traditionally associated with vehicle design, we found accessibility as a critical value for our target user group. We also identified emerging conflicts between safety and the values of mobility, freedom, or privacy in multiple planned vehicle functions. The results will serve as a basis for an increased stakeholder involvement in the development of autoELF and an expansion of functional requirements. We expect the systematic consideration of the stakeholders' values to result in an improved vehicle concept and prototype at the end of the project in 2022. In the future, we will further explore how formal technical requirements and system design specifications can address uncovered values and emerging value conflicts. 


\section{Acknowledgement}

This research is accomplished within the project "UNICARagil" (FKZ 16EMO0285). We acknowledge the financial support for the project by the Federal Ministry of Education and Research of Germany (BMBF). We thank our colleagues Susanne Ernst and Marcus Nolte for introducing Value Sensitive Design to us as part of their work is in the project "Value-Based Decision Making" funded by the Daimler and Benz Foundation. Additionally, we thank Sonja Luther for proofreading and all lab members - particularly Inga Jatzkowski and Torben Stolte - for helpful discussions regarding the vehicle project used in this paper as a case study.

\section{References}

Bubb, H. (2007), "Future Applications of DHM in Ergonomic Design", In: Duffy, V.G. (Ed.), Digital Human Modeling, Lecture Notes in Computer Science 4561, Springer, Berlin Heidelberg, Germany, pp. 779-793. https://doi.org/10.1007/978-3-540-73321-8_89

Cawthorne, D. and Cenci, A. (2019), "Value Sensitive Design of a Humanitarian Cargo Drone", 2019 International Conference on Unmanned Aircraft Systems (ICUAS), Atlanta, GA, USA, IEEE, Piscataway, NJ, USA, pp. 11171125. https://doi.org/10.1109/ICUAS.2019.8797940

Davis, J. and Nathan, L.P. (2015), "Value Sensitive Design: Applications, Adaptations, and Critiques", In: van den Hoven, J., Vermaas, P.E. and van de Poel, I. (Eds.), Handbook of Ethics, Values, and Technological Design, Springer, Dordrecht, The Netherlands, pp. 11-40. https://doi.org/10.1007/978-94-007-6970-0_3

Friedman, B., Hendry, D.G. and Borning, A. (2017), "A Survey of Value Sensitive Design Methods", Foundations and Trends in Human-Computer Interaction, Vol. 11 No. 2, pp. 63-125. https://doi.org/10.1561/1100000015

Friedman, B. et al. (2013), "Value Sensitive Design and Information Systems", In: Doorn, N., Schuurbiers, D., van de Poel, I. and Gorman, M.E. (Eds.), Early Engagement and New Technologies: Opening Up the Laboratory, Philosophy of Engineering and Technology 16, Springer, Dordrecht, The Netherlands, pp. 55-95. https://doi.org/10.1007/978-94-007-7844-3_4

Friedman, B., Kahn, P.H. and Howe, D.C. (2000), "Trust online", Communications of the ACM, Vol. 43 No. 12, pp. 34-40. https://doi.org/10.1145/355112.355120

Gerdes, J.C., Thornton, S.M. and Millar, J. (2019), "Designing Automated Vehicles Around Human Values", In: Meyer, G. and Beiker, S. (Eds.), Road Vehicle Automation 6, Lecture Notes in Mobility, Springer, Cham, Switzerland, pp. 39-48. https://doi.org/10.1007/978-3-030-22933-7_5

Graubohm, R. et al. (2017), "Systematic Design of Automated Driving Functions Considering Functional Safety Aspects", 8. Tagung Fahrerassistenz, Munich, Germany, Chair of Automotive Technology with TÜV SÜD Academy, Garching, Germany.

Halsey, A. (2017), Driverless cars promise far greater mobility for the elderly and people with disabilities. [online] The Washington Post. Available at: https://www.washingtonpost.com/local/trafficandcommuting/driverless-carspromise-far-greater-mobility-for-the-elderly-and-people-with-disabilities/2017/11/23/6994469c-c4a3-11e7-84bc5e285c7f4512_story.html (accessed 11.11.2019).

Hoff, K.A. and Bashir, M. (2015), "Trust in Automation: Integrating Empirical Evidence on Factors That Influence Trust", Human Factors, Vol. 57 No. 3, pp. 407-434. https://doi.org/10.1177/0018720814547570

Lee, J.D. and See, K.A. (2004), "Trust in Automation: Designing for Appropriate Reliance”, Human Factors, Vol. 46 No. 1, pp. 50-80. https://doi.org/10.1518/hfes.46.1.50_30392

Pfleging, B., Rang, M. and Broy, N. (2016), "Investigating User Needs for Non-Driving-Related Activities During Automated Driving", Proceedings of the 15th International Conference on Mobile and Ubiquitous Multimedia (MUM '16), Rovaniemi, Finland, ACM, New York, NY, USA, pp. 91-99. https://doi.org/10.1145/3012709. 3012735

Schräder, T. et al. (2019), "An Approach for a Requirement Analysis for an Autonomous Family Vehicle”, 2019 IEEE Intelligent Vehicles Symposium (IV), Paris, France, IEEE, Piscataway, NJ, USA, pp. 1597-1603. https://doi.org/10.1109/IVS.2019.8813882

Thornton, S.M. (2018), Autonomous vehicle motion planning with ethical considerations, [PhD Thesis], Stanford University.

Thornton, S.M. et al. (2018), "Value Sensitive Design for Autonomous Vehicle Motion Planning", 2018 IEEE Intelligent Vehicles Symposium (IV), Changshu, China, IEEE, Piscataway, NJ, USA, pp. 1157-1162. https://doi.org/10.1109/IVS.2018.8500441

Winkler, T. and Spiekermann, S. (2018), "Twenty years of value sensitive design: a review of methodological practices in VSD projects", Ethics and Information Technology. https://doi.org/10.1007/s10676-018-9476-2

Woopen, T. et al. (2018), "UNICARagil - Disruptive Modular Architectures for Agile, Automated Vehicle Concepts", 27th Aachen Colloquium Automobile and Engine Technology 2018, Aachen, Germany, Aachener Kolloquium Fahrzeug- und Motorentechnik GbR, Aachen, Germany, pp. 663-694. https://doi.org/10.18154/RWTH-2018229909 